Reflexión

\title{
LA TECNOLOGÍA EDUCATIVA EN EL PROCESO DE ENSEÑANZA- APRENDIZAJE EN LA ENFERMERÍA DESDE UN ANÁLISIS DE LA TEORÍA INTERACCIONISTA DE VYGOTSKI
}

\author{
EDUCATIONAL TECHNOLOGY IN THE TEACHING-LEARNING PROCESS IN \\ NURSING FROM AN ANALYSIS OF THE INTERACTIONAL THEORY OF \\ VYGOTSKI
}

\section{Barbara Caroliny Pereira}

Mestranda do Programa de Pós Graduação em Enfermagem. Universidade Federal de Alfenas (UNIFAL-MG), Brasil.

\section{Lara Aparecida de Freitas}

Universidade Federal de Alfenas (UNIFAL-MG), Brasil.

\section{Silvana Maria Coelho Leite Fava}

Universidade Federal de Alfenas (UNIFAL-MG), Brasil.

\section{Simone Albino da Silva}

Universidade Federal de Alfenas (UNIFAL-MG), Brasil.

\section{Zélia Marilda Rodrigues Resck}

Universidade Federal de Alfenas (UNIFAL-MG), Brasil.

Artículo recibido el 11 de junio de 2018. Aceptado en versión corregida el 7 de noviembre de 2018.

\section{RESUMEN}

Objetivo: analizar el uso de tecnología como estrategia en el proceso de enseñanzaaprendizaje en la enfermería a la luz de la Teoría Interaccionista de Vygotski. Método: Estudio teórico-reflexivo, elaborado a partir de una Revisión Narrativa, se lanzó como referencial el modelo de aprendizaje de Vygotski, Teoría Interaccional. Resultados: Se identificaron 11 artículos que atendieron los criterios de inclusión y fueron codificados en la siguiente categoría: "Tecnología como estrategia de enseñanza-aprendizaje en la enfermería a la luz de la Teoría Interaccionaria de Vygotski", en la que posibilitó discutir y reflexionar sobre la docencia, práctica clínica y actuación de la enfermería frente a la tecnología. Conclusión: La literatura trae en relación al proceso enseñanza-aprendizaje a la luz de la teoría interaccionista de Vygotski la importancia de la presencia del profesor como mediador/facilitador y las tecnologías como complemento para facilitar el aprendizaje y posibilitar una mayor interacción entre alumnos y alumnos-profesores. 
Palabras clave: Tecnología Educativa, Informática en Enfermería, Educación en Enfermería, Tecnología de la información, Enfermería.

\begin{abstract}
Objective: to analyze the use of technology as a strategy in the teaching-learning process in nursing in light of Vygotsky's Theory of Interaction. Method: Theoretical-reflexive study, elaborated from a Narrative Review, Vygotsky's learning model, Interaccional Theory was launched as a reference. Results: Eleven articles were identified that met the inclusion criteria and were codified in the following category: "Technology as a teaching-learning strategy in nursing in the light of Vygotsky's Interactional Theory", in which it was possible to discuss and reflect on teaching, clinical practice and nursing action in front of technology. Conclusion: Literature brings in relation to the teaching-learning process in light of the interactionist theory of Vygotsky the importance of the presence of the teacher as mediator / facilitator and technologies as a complement to facilitate learning and enable greater interaction between students and students -teachers.
\end{abstract}

Keywords: Educational Technology, Informatics in Nursing, Nursing Education, Information Technology, Nursing.

\title{
http://dx.doi.org/10.7764/Horiz_Enferm.29.3.251-262
}

\section{INTRODUCCIÓN}

La Revolución Industrial del siglo XVIII fue un hito en el uso del término "tecnología", el que fue generalizado para ser aplicado a diferentes áreas de conocimiento, además que en los sectores de la industria textil y mecánica. De esta manera, la tecnología surgió como forma de facilitar la vida humana y sus tareas, ya que la sociedad se hizo más tecnológica, también en la educación, por medio de la utilización de estrategias pedagógicas activas en el proceso de enseñanzaaprendizaje $^{(1)}$.

El Diccionario de la Lengua Portuguesa, de Antônio Houaiss, indica la palabra "tecnología" como "un conjunto de conocimientos científicos, de los procesos y métodos usados en la creación y en la utilización de bienes y servicios"
A lo largo de los años, Brasil evolucionó en relación a la informatización y todo esto contribuyó para el desarrollo de la práctica clínica, y, consecuentemente elevó la producción en el área de la educación en enfermería.

Hubo entonces un creciente avance en el uso de estas tecnologías para la enfermería, con multiplicidad de temas abordados, impacto del Internet, enfoque en el postgrado, desarrollo de competencias y auxilio en la toma de decisiones $^{(2)}$.

En la actualidad, ha ocurrido el desarrollo de estrategias de enseñanza, mediante las tecnologías en el campo de la enfermería, en su mayoría vinculado a las universidades, sea esto en la educación en salud o en proyectos que contribuyan en la formación profesional ${ }^{(3)}$. En cuanto a la 
Pereira, B.C., de Freitas,L.A., Coelho Leite Fava, S.M., da Silva, S.A., Rodrigues Resck, Z.M.

trayectoria histórica, la utilización de la informática en la enfermería se hizo presente desde la década de los 60 en los Estados Unidos de América, Alemania, Japón, Francia y Finlandia, principalmente en lo que se dice sobre la gestión en enfermería $^{(2)}$.

La globalización es un proceso ocurrido desde el siglo XX, en el cual hubo un aumento de interacción entre países y personas, y todo esto también hizo que se ocasionaran cambios en la educación, y principalmente, en la enseñanza superior. Dado que la tecnología de la información provoca transformaciones en la vida de las personas, así como está inserida en la sociedad y en el medio académico, el uso de la tecnología en la educación ha sido el camino para contribuir en la formación de los profesionales, la cual puede proporcionar creatividad para enfrentar los desafíos de la sociedad, adaptación al mercado de trabajo con capacitación y favorecimiento en las prácticas de salud ${ }^{(4)}$.

Las relaciones humanas han sido modificadas a lo largo de los años, así como el comportamiento humano, las relaciones familiares y profesionales. Las nuevas tecnologías han aumentado en los grandes centros urbanos, así como el acceso a los medios de comunicación, lo que antes era restricto a una determinada población, y todo esto contribuyó para cambios de comportamiento, llevando a falta de tiempo a las personas ${ }^{(1)}$.

Con este avance, el Internet pasó a marcar esta era tecnológica, se estima que prácticamente el 90\% de los jóvenes de las regiones más pobladas de Brasil ya posean el Internet en casa. Apenas la región Norte presenta un índice menor ${ }^{(5)}$. Se puede decir que en estos días donde la tecnología y el
Internet son las formas más atractivas de comunicación para los jóvenes y hasta mismo para muchos adultos, hacerles aceptar y aprovechar un contenido de aula de forma expositiva ciertamente será con dificultades, ya que el joven está acostumbrado a interactuar todo el tiempo y a buscar informaciones en el Internet ${ }^{(6)}$.

El pasar de los años ha proporcionado esta transición de la Era de la Información para la Era de Conocimiento, en la cual se traduce en ampliación de la interacción entre alumnoprofesor y alumno-alumno, nuevos formatos en el proceso de enseñanzaaprendizaje, en la interacción social, entre otros $^{(1)}$. Transición que se debe a la descentralización del conocimiento exclusivo del profesor, y al aumento del empoderamiento en el desarrollo y en la capacidad del alumno por medio de recursos interactivos, como las innovaciones tecnológicas.

Cada vez más los jóvenes se mantienen ligados y conectados a Internet en su mayor parte del tiempo, y esto favorece un contenido significativo que para el alumno se ha hecho más difícil, ya que es necesario mucho más para llamar la atención delante de múltiplos dados que el mismo accede. Frente a esto, se hace necesario que los docentes se apropien de los recursos tecnológicos y los utilicen como estrategias en el proceso de enseñanza-aprendizaje. De este modo, el objetivo del estudio fue analizar el uso de la tecnología como estrategia en el proceso de enseñanza aprendizaje en la enfermería a la luz de la Teoría Interaccionista de Vygotski. 


\section{MÉTODO}

Estudio

teórico-reflexivo, elaborado a partir de una Revisión Narrativa realizada en septiembre de 2017. Como estrategias de busca se emplearon los Descriptores en Ciencia de la Salud (DeCS) y los Medical Subject Headings (MeSH) y los operadores booleanos AND y OR; los criterios de elegibilidad incluyeran: artículos con disponibilidad gratuita en su totalidad, de los últimos cinco años y en las lenguas portuguesa, inglesa y española. Fueran excluidos los artículos que no satisfacían estos criterios y aquellos que no abordaban la temática del estudio.

La búsqueda fue realizada en la Base de Dados Medical Literature Analysis and Retrieval System Online (MEDLINE), en la Literatura LatinoAmericana en Ciencias de la Salud (LILACS) y en la Base de Dados de Enfermería (BDENF) y se utilizaron los descriptores "Tecnología educacional", "Informática en enfermería", "Tecnología de la información", "Educación en enfermería" y "Enfermería" en las respectivas bases.

Además, para un mejor embasamiento, se lanzó como referencial el modelo de aprendizaje de Vygotski, Teoría Interaccionista, en la cual las interacciones son una forma de adquisición del conocimiento, junto con las acciones de los sujetos que están inseridos en el proceso de aprendizaje. El alumno es visto como propio constructor de su conocimiento, siendo determinado por una sociedad y cultura.
La tecnología educativa en el proceso....

\section{RESULTADOS}

Fueran identificados 11 artículos que atenderán los criterios de inclusión. Teniendo como objetivo lo de posibilitar una mayor comprensión y discusión, los artículos fueran relacionados con la teoría de Vygotski en la categoría: "Tecnología como estrategia de enseñanza-aprendizaje en la enfermería a la luz de la Teoría Interaccionista de Vygotski” El cuadro 1 ilustra los artículos seleccionados en la muestra con títulos, autores, año de publicación y objetivo.

\section{Cuerpo de la reflexión}

\section{Tecnología como estrategia de enseñanza-aprendizaje en la enfermería a la luz de la Teoría Interaccionista de Vygotski}

Lev Semyonovich Vygotsky fue un psicólogo, proponente de la Psicologia cultural-histórica, que dedicó sus estudios a diversas áreas de conocimiento. Investigó y reflexionó sobre cuestiones de la Psicología, de la Filosofía, de la Literatura, de la Educación, del Derecho, de las Artes, de la Medicina. En 1919, Vygotsky pasó a actuar como profesor de lengua y literatura rusa, lógica, psicología, historia, ética y filosofía. Su primera grande obra, La psicología pedagógica, trata de su experiencia como docente ${ }^{(7)}$.

En la teoría interaccionista de Vygotsky el hombre es visto como alguien que transforma y es transformado por las relaciones sociales. Lo que ocurre es una interacción (dialéctica) entre el ser 
Pereira, B.C., de Freitas,L.A., Coelho Leite Fava, S.M., da Silva, S.A., Rodrigues Resck,

Cuadro 1. Distribución de los artículos seleccionados: títulos, autores, año de publicación y objetivo. Alfenas (MG), Brasil, 2017. Fuente: autores, 2017.

\begin{tabular}{|c|c|c|c|}
\hline Título & Autores & Año & Objetivos \\
\hline $\begin{array}{l}\text { Desarrollo de recursos } \\
\text { educacionales digitales } \\
\text { para la enseñanza en } \\
\text { enfermería. }\end{array}$ & $\begin{array}{l}\text { Tibes CM, Dias JD, } \\
\text { Westin, UM, } \\
\text { Domingues, AN, } \\
\text { Zem-Mascarenhas } \\
\text { SH, Évora YDM. }\end{array}$ & 2017 & $\begin{array}{l}\text { Desarrollar recursos educacionales } \\
\text { utilizando la metodología } \\
\text { WebQuest para auxiliar en el } \\
\text { proceso de enseñanza y aprendizaje } \\
\text { de estudiantes y profesionales de } \\
\text { Enfermería. }\end{array}$ \\
\hline $\begin{array}{l}\text { Plataforma Moodle en } \\
\text { la construcción del } \\
\text { conocimiento en } \\
\text { Terapia Intensiva: } \\
\text { estudio experimental }\end{array}$ & $\begin{array}{l}\text { Domenico EBLD, } \\
\text { Cohrs CR. }\end{array}$ & 2016 & $\begin{array}{l}\text { Comparar el perfeccionamiento de } \\
\text { conocimientos y habilidades de los } \\
\text { graduandos que participaron a las } \\
\text { actividades propuestas en el } \\
\text { Ambiente Virtual de Aprendizaje, } \\
\text { Moodle, en el periodo de practica } \\
\text { Hospitalar en unidad de terapia } \\
\text { intensiva. }\end{array}$ \\
\hline 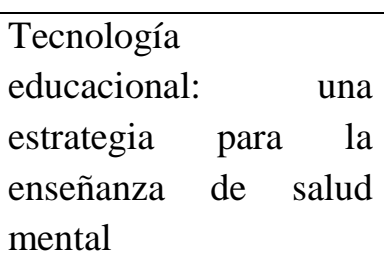 & $\begin{array}{l}\text { Botti NCL, Mesquita } \\
\text { IR, Pereira CCM, } \\
\text { Araújo FA. }\end{array}$ & 2015 & $\begin{array}{l}\text { Describir el proceso de desarrollo y } \\
\text { validación del software educativo } \\
\text { Quiz Loucura desarrollado por ser } \\
\text { utilizado como recurso didáctico en } \\
\text { la enseñanza de salud mental. }\end{array}$ \\
\hline $\begin{array}{l}\text { Un trabajo de } \\
\text { investigación-acción } \\
\text { con uso de metodología } \\
\text { activa en la enseñanza } \\
\text { de tecnología de la } \\
\text { información. }\end{array}$ & $\begin{array}{l}\text { Gouvea EP, Odagima } \\
\text { AM, Shitsuka DM, } \\
\text { Shitsuka R. }\end{array}$ & 2015 & $\begin{array}{l}\text { Presentar un estudio sobre el } \\
\text { proceso de enseñanza-aprendizaje } \\
\text { para alumnos de Tecnología de la } \\
\text { Información, utilizando la } \\
\text { metodología de la investigación- } \\
\text { acción. }\end{array}$ \\
\hline $\begin{array}{l}\text { Tecnología y } \\
\text { metodología para una } \\
\text { educación sin distancia. }\end{array}$ & & 2015 & $\begin{array}{l}\text { Hace un análisis sobre el futuro de } \\
\text { la sala de aula, sea esta concreta o } \\
\text { virtual, a la luz del perfil de las } \\
\text { nuevas generaciones, y de las } \\
\text { tendencias tecnológicas en la } \\
\text { educación. }\end{array}$ \\
\hline $\begin{array}{lrr}\text { Objeto } & \text { digital } & \text { en } \\
\text { enfermería } & \text { neonatal: } \\
\text { impacto } & \text { en } & \text { el } \\
\text { aprendizaje } & & \text { de } \\
\text { estudiantes. } & & \end{array}$ & $\begin{array}{ll}\text { Aredes NDA, Góes } \\
\text { FSN, Silva } & \text { MAI, } \\
\text { Gonçalves } & \text { MFC, } \\
\text { Fonseca LMM. }\end{array}$ & 2015 & $\begin{array}{l}\text { Comparar el aprendizaje cognitivo } \\
\text { de estudiantes de enfermería, sobre } \\
\text { evaluación clínica del prematuro, } \\
\text { dividiendo los entre los que } \\
\text { utilizaron y que no utilizaron el } \\
\text { objeto digital de aprendizaje (ODA) } \\
\text { como material de apoyo. }\end{array}$ \\
\hline $\begin{array}{l}\text { Uso y desarrollo de } \\
\text { tecnologías para la }\end{array}$ & $\begin{array}{l}\text { Salvador } \\
\text { Rodrigues }\end{array}$ & 2015 & $\begin{array}{l}\text { Caracterizar las tecnologías para la } \\
\text { enseñanza utilizadas }\end{array}$ \\
\hline
\end{tabular}


La tecnología educativa en el proceso....

\begin{tabular}{|l|l|l|l|}
\hline $\begin{array}{l}\text { enseñanza presentados } \\
\text { en investigación de } \\
\text { enfermería. }\end{array}$ & $\begin{array}{l}\text { Lima KYN, Alves } \\
\text { KYA, Santos VEP. }\end{array}$ & $\begin{array}{l}\text { desarrolladas en las disertaciones y } \\
\text { tesis de enfermería de Brasil. }\end{array}$ \\
\hline $\begin{array}{l}\text { Tecnologías educación } \\
\text { educacionales digitales } \\
\text { para } \\
\text { profesional de nivel } \\
\text { medio en enfermería. }\end{array}$ & $\begin{array}{l}\text { Góes, FSN, Camargo, } \\
\text { RAA, Hara CYN, } \\
\text { Fonseca LMM. }\end{array}$ & 2014 & $\begin{array}{l}\text { Identificar lo que se ha producido } \\
\text { sobre el uso de tecnologías } \\
\text { educacionales digitales para } \\
\text { educación profesional de nivel } \\
\text { medio en enfermería. }\end{array}$ \\
\hline $\begin{array}{l}\text { El uso de los } \\
\text { dispositivos móviles en } \\
\text { el proceso de enseñanza } \\
\text { y aprendizaje en el } \\
\text { medio virtual }\end{array}$ & $\begin{array}{l}\text { Saboia J, Viva MAA, } \\
\text { Vargas PL. }\end{array}$ & 2013 & $\begin{array}{l}\text { Discursar sobre el uso de las } \\
\text { tecnologías móviles, presentando } \\
\text { los tipos de dispositivos móviles } \\
\text { existentes, direccionando para la } \\
\text { construcción del entendimiento del } \\
\text { uso de tales criterios junto al perfil } \\
\text { del alumno encontrado en cursos de } \\
\text { educación a distancia. }\end{array}$ \\
\hline $\begin{array}{l}\text { Recursos tecnológicos } \\
\text { en la educación en } \\
\text { enfermería. }\end{array}$ & $\begin{array}{l}\text { Tobase L, Guareschi } \\
\text { APDF, Frias MAE, } \\
\text { Prado C, Peres HHC. }\end{array}$ & 2013 & $\begin{array}{l}\text { Identificar la utilización de los } \\
\text { recursos tecnológicos en la } \\
\text { educación en enfermería. }\end{array}$ \\
\hline $\begin{array}{l}\text { El internet y su } \\
\text { influencia en el proceso } \\
\text { enseñanza aprendizaje } \\
\text { de estudiantes de } \\
\text { enfermería. }\end{array}$ & $\begin{array}{l}\text { Leite, KNS, Santos, 2013 } \\
\text { SR, Andrade, SSC, } \\
\text { Zaccara, AAL, Costa, } \\
\text { TF. }\end{array}$ & $\begin{array}{l}\text { Identificar las herramientas del } \\
\text { internet más utilizadas por } \\
\text { estudiantes de enfermería y } \\
\text { describir como perciben su } \\
\text { influencia en el proceso enseñanza- } \\
\text { aprendizaje. }\end{array}$ \\
\hline
\end{tabular}

humano y el medio social y cultural donde está inserido. Como seres humanos, necesitamos vivir en convivio social, pues somos considerados seres sociales y por lo tanto, podemos establecer relaciones personales, adquirir conocimientos que van a posibilitar la construcción de un ser humano protagonista de du historia ${ }^{(8)}$.

Se puede decir que los Modelos pedagógicos son sustentados por las Teorías de Aprendizaje, siendo una fundamentación direccionadora de como será articulado el modelo, los procedimientos y las técnicas para la promoción de aprendizaje ${ }^{(9)}$.

Se debe acrecentar que la construcción del conocimiento por el abordaje vygotskyano ocurre por la interacción del sujeto con el ambiente sociocultural donde vive, o sea, ningún conocimiento es construido por la persona solita, sino por las interacciones de la misma con el grupo. La educación debe, en esta perspectiva, tomar como referencia toda la experiencia de vida propia del sujeto $^{(9)}$.

Vygotski afirma que el proceso de construir conocimiento pasa por una acción compartida, lo que implica un proceso de mediación entre sujetos. El profesor actúa como mediador y el aprendizaje está en la observación del medio y en la discusión y organización de las descubiertas conjuntamente entre 
Pereira, B.C., de Freitas,L.A., Coelho Leite Fava, S.M., da Silva, S.A., Rodrigues Resck, Z.M.

alumnos y profesores. En esta perspectiva, la interacción social es la condición indispensable para el aprendizaje ${ }^{(8)}$.

Vygotsky complementa afirmando que la mediación ocurre además de la interacción entre las personas y el ambiente, y sucede también por medio del uso de instrumentos, o sea, de los signos. Estos signos, según él, son sistemas simbólicos, criados por la sociedad a lo largo del tiempo, que permiten a las personas de cambiar sus niveles de desarrollo cultural y la forma social ${ }^{(10)}$.

Esta interacción se hace efectiva a partir del momento en el cual existe una comunicación entre los involucrados, que pueden compartir pensamientos y acciones opuestas o parecidas, que se convierten a partir de ese momento, en piezas clave y responsables por el proceso de aprendizaje.

En esta perspectiva de construcción del conocimiento, la enseñanza debe tratar de proporcionar en el alumno un pensamiento innovador $\mathrm{y}$ crítico, en la cual se crean ideas y no se realizan copias de lo que los otros ya hicieron, o sea, se crean nuevas formas de conocimiento $^{(6,11)}$. Esto hace posible que el alumno se haga activo, productivo, capaz de recibir el conocimiento y de elaborar reflexiones a partir del mismo, utilizando la tecnología como estrategia de enseñanza-aprendizaje. El objetivo es que la enseñanza ultrapase los limites puramente técnicos, para alcanzar efectivamente el cuidado y la aprensión de lo que se quiere enseñar.

Existe una concordancia en decir que los jóvenes de hoy en día están desinteresados y apáticos en relación a los estudios y a la búsqueda de conocimiento.
Cabe al profesor promover esta mejora en el aprendizaje o apenas descubrir los verdaderos motivos de esta desmotivación, con una reflexión personal y continua sobre el aprendizaje ${ }^{(12)}$. Los estudiantes deben adquirir conocimientos y habilidades de forma que sean capaces de tomar decisiones, principalmente en el cuidado en la prestación de la asistencia de enfermería a los pacientes ${ }^{(13)}$.

Una de las ventajas del uso de herramientas tecnológicas consiste en ofrecer al alumno una fácil comprensión del asunto abordado ${ }^{(14)}$. Por lo tanto, su uso puede proporcionar un conocimiento bilateral, entre varias personas al mismo tiempo y de forma que todos puedan entender.

La tecnología en la educación permite la flexibilización de la enseñanza, haciéndolo más dinámico, actual y accesible $^{(15)}$. Posibilita al alumno una construcción reflexiva del conocimiento y atender las necesidades del contexto en el cual el alumno se desarrolla.

Se destaca que el aprendizaje colectivo auxilia en el proceso de enseñanza, basado en desafíos y problemas, pero respectando el ritmo de cada uno ${ }^{(16)}$. El conocimiento cuando es abordado y diseminado en grupos, puede alcanzar niveles mayores, aumentando más allá de las relaciones entre los involucrados, la interacción entre el conocimiento recibido y el conocimiento transmitido, con un enfoque en la colectividad.

El proceso de aprendizaje según Vygotski va tanto en dirección ascendente como descendente. En la ascendencia, el vector indica la acción de los conceptos espontáneos, abriendo el camino para los 
conceptos científicos, mientras, en la descendencia, indica la influencia científica sobre el conocimiento cotidiano, fortaleciendo las estructuras para el desarrollo ascendente del mismo, siempre en una relación dialéctica ${ }^{(17)}$.

Para Vygotski, el desarrollo de los lenguajes de computación es la interdependencia entre el lenguaje y el pensamiento. Las ciencias cognitivas actualmente se apoyan en las ideas vygotskianas para la elaboración de nuevas y más eficientes estrategias de enseñanza/aprendizaje. Las ciencias de computación se basan en la premisa que el lenguaje y el pensamiento se moldean mutuamente ${ }^{(18)}$.

En esta premisa, se verifica que los jóvenes de la modernidad tienen más facilidad en el proceso de enseñanzaaprendizaje cuando les es ofrecido con la tecnología, la cual posibilita una relación positiva de interacción en grupos, basada en intercambio de experiencias y aprendizaje colaborativo $^{(14)}$.

Sin embargo, se puede reflexionar sobre puntos positivos y negativos en el uso de la tecnología, o sea, el internet, así como los diversos medios de comunicación, antes restitos, que se hicieron un medio de alejamiento entre las personas, reflexionando en cambios en la vida familiar. Los comportamientos se hicieron menos afectivos, las relaciones más distantes y las personas menos unidas. Pero, el uso de las tecnologías contribuyó mucho para el crecimiento y la propagación del conocimiento, donde una información cuando divulgada rápidamente es capaz de alcanzar horizontes inmensurables, pudiendo aun ser transformadora y completamente modificable en fracciones de minutos.

La teoría de Vygotski tiene su origen a partir de las inter-relaciones del individuo y su contexto social y cultural. La cultura es parte de la naturaleza humana, pues el desarrollo mental humano no es algo pasivo, ocurre a partir de la actividad práctica, es participando activamente en la colectividad, que el individuo se apropia del lenguaje. El aprendizaje es un proceso continuo ${ }^{(19,20)}$.

Con esto, se ve que la enseñanza no puede ser realizada de forma estandardizada, aunque el profesor tiene el dominio del conocimiento, requiere una planificación, pues cada grupo es único y cada proceso de aprendizaje funciona de una forma para determinados alumnos. Para estos fines, el proceso de enseñanzaaprendizaje debe ser contextualizado de acuerdo con el grupo de alumnos en la cual irá a actuar, ya que la formación profesional ocurre de manera críticareflexiva, en los espacios de interacción y por la mediación de los profesores ${ }^{(21)}$.

La aproximación con la literatura permitió reflexionar sobre el facto que mismo con el avance de la tecnología es notorio que muchos profesores y alumnos aun presentan dificultades y barreras al lidiar con las herramientas tecnológicas en la educación, pero, ptors presentan facilidades. De este modo, la interacción de alumnos y profesores con diferentes experiencias, la forma mutua y compartida en la construcción del conocimiento puede favorecer el aprendizaje por medio del uso de la tecnología.

La enseñanza debe ser capaz de proporcionar al alumno su auto 
Pereira, B.C., de Freitas,L.A., Coelho Leite Fava, S.M., da Silva, S.A., Rodrigues Resck, Z.M.

aprendizaje también, hacerlo más activo y constructivo, capaz de proponer hipótesis $y$ formular estrategias interactivas $y$ participativas. No solamente en el campo académico, también en el ambiente de trabajo y en toda la parte clínica ${ }^{(21)}$.

Para Vygotski, el lenguaje y los pensamientos caminan juntos. Según se aprende a usar, efectivamente, la función planificadora del lenguaje, el campo psicológico cambia radicalmente. La capacitación humana para el lenguaje habilita a providenciar instrumentos auxiliadores en la solución de tareas difíciles, a superar la acción impulsiva, a planear una solución para un problema antes de su ejecución y a controlar su propio comportamiento. Se señala que las funciones cognitivas y comunicativas del lenguaje es lo que distingue las personas de los animales, pues se hacen la base de una forma nueva y superior de actividad (18).

Vygotski ve la asimilación como algo activo. En el caso de los adultos el proceso de memorización ocurre en ausencia de auxiliares externos especiales, pues se encuentra completamente desarrollado ${ }^{(18)}$.

Vygotski atribuye como proceso de memorización y aprendizaje cuando una persona ata un nodo en un pañuelo para ayudarse a recordar de algo, haciendo con que el objeto externo la recuerde; ella transforma el proceso de recuerdo en una actividad externa. Afirma que son los signos que ayudan los seres humanos a recordarse activamente de las cosas, es donde se encuentra la verdadera esencia de la memoria humana ${ }^{(22)}$.

Es posible inferir que los recursos tecnológicos sean considerados signos en favorecimiento de su memorización que auxilian en el proceso de enseñanzaaprendizaje, considerando que el alumno es activo y constructor de su conocimiento. Vale la pena acrecentar la importancia del papel del profesor como mediador de este proceso.

El proceso de enseñanzaaprendizaje puede ser comprendido de forma mejor cuando se hace referencia al concepto de Zona de Desarrollo Proximal (ZDP). Para Vygotski la ZDP es la distancia entre el nivel de desarrollo real, o sea, determinado por la capacitad de resolver problemas independientemente, y el nivel de desarrollo proximal, demarcado por la capacidad de solucionar problemas con la ayuda de un compañero más experto. Es justamente en esta zona de desarrollo proximal que el aprendizaje va a ocurrir ${ }^{(22)}$. Entender la zona de desarrollo proximal es la base para la personalización de la educación, hoy viabilizada por los recursos digitales.

Corroborando con la Teoría, se ve la importancia de una remodelación en el proceso de formación de los profesionales de enfermería, que necesitan de un incentivo para el raciocinio clínico, la valorización de la articulación entre teoría y práctica, la utilización de metodologías activas en el proceso de enseñanzaaprendizaje, la flexibilidad curricular y el reconocimiento de un equipo multidisciplinar en la práctica profesional $^{(23)}$. Se abre una variedad de opciones para amplias innovaciones en la enseñanza por medio de herramientas tecnológicas, con una enseñanza activa y diferenciada, innovando también los papeles de los actores del proceso. 
La tecnología educativa en el proceso....

Estos conceptos de Vygotski, además de contribuir para el proceso de enseñanza-aprendizaje de los alumnos, haciéndose activos, reflexivos y constructivos, también son de suma importancia para que el enfermero aplique esta Teoría en su práctica clínica, haciendo que su equipo sea activo y reflexivo. Es una forma de crear medios de interacción con el equipo, los pacientes y los familiares.

El enfermero, en el enfoque vygotskiana, también tiene el papel de educador, o sea, de promover el desarrollo de su equipo a través de acciones educativas, proporcionando siempre la actualización del equipo de enfermería ${ }^{(20)}$. Vale resaltar que la informatización dentro de la enfermería no se refiere apenas a ordenadores, pero a una forma de innovación, de hacer de las herramientas tecnológicas algo a ser aplicado dentro del equipo multiprofesional, que permita la práctica holística y más efectiva.

La enfermería no ve la tecnología como un arte, pero la utiliza para favorecer el proceso de enseñanza-aprendizaje, el proceso de trabajo y la calidad de su asistencia, favoreciendo un cuidado amplio y digno a los pacientes, además de promover la diseminación del conocimiento, sin la intención de substituir la interacción de las relaciones humanas ${ }^{(2)}$.

El desafío en el proceso de enseñanza-aprendizaje está en el hacer uso de los signos para diseminar este conocimiento y de utilizar todos los tipos posibles de tecnología para tal acción. Se hace necesario que docentes y profesionales vean la tecnología como una herramienta posible para abrir una gama de posibilidades para el proceso de enseñanza-aprendizaje.

\section{CONCLUSIONES}

Es posible remeter por la teoría interaccionista de Vygostsky que la utilización de los recursos tecnológicos en el proceso de enseñanza-aprendizaje constituyen signos importantes. Estos pueden favorecer el aprender y el enseñar, por la mediación del profesor y por la interacción entre profesores y alumnos por medio del intercambio de experiencias y vivencias. En este contexto, el uso de la tecnología en la educación en enfermería puede propiciar la formación de profesionales proactivos, críticos y reflexivos, además de estimular el aprendizaje continuo y actualizado, favoreciendo la educación permanente.

Con este, la literatura trae en relación al proceso enseñanza-aprendizaje a la luz de la teoría interaccionista de Vygostsky, la importancia de la presencia del profesor como mediador/facilitador, y las tecnologías como complemento para facilitar el aprendizaje y posibilitar una mayor interacción entre alumnos y alumnos-profesores.

Nuevos estudios son sugeridos, para que la tecnología se pueda constituir como estrategia de enseñanza-aprendizaje en diferentes áreas del conocimiento, en la formación de enfermeros críticosreflexivos y de profesionales competentes.

\section{REFERENCIAS BIBLIOGRÁFICAS}

1. Saboia J, Viva MAA, Vargas PL. O uso dos disposítivos móveis no processo de ensino e aprendizagem no meio virtual. 
Pereira, B.C., de Freitas,L.A., Coelho Leite Fava, S.M., da Silva, S.A., Rodrigues Resck, Z.M.

Revista Cesuca Virtual:Conhecimento sem fronteiras. 2013 Julho 1(1).

2. Tobase L, Guareschi APDF, Frias MAE, Prado C, Peres HHC. Recursos tecnológicos na educação em enfermagem. J. Health Inform. 2013; 5(3): 77-81.

3. Botti NCL, Mesquita IR, Pereira CCM, Araújo FA. Tecnologia educacional: uma estratégia para o ensino de saúde mental. Cadernos Brasileiros de Saúde Mental, 2015; Florianópolis; 7 (15): 24-31.

4. Leite, KNS, Santos, SR, Andrade, SSC, Zaccara, AAL, Costa, TF. The internet and its influence in learning-teaching process of nursing students. Rev. enferm. UERJ, Rio de Janeiro, 2013 out/dez; 21(4):46470.

5. Passarelli B, Junqueira AH. Gerações Interativas Brasil: Crianças e Adolescentes Diante das Telas. São Paulo: Escola do Futuro/USP, 2012.

6. Gouvea EP, Odagima AM, Shitsuka DM, Shitsuka R. Um trabalho de pesquisa-ação com uso de metodologia ativa no ensino de tecnologia da informação. Educação, Gestão e Sociedade, 2015; 5(20).

7. Prochnow ALC, Vieira ANG, Marchesan MTN. O interacionismo social de Vigotski e o constructo de crenças sobre ensino e aprendizagem de línguas. SCRIPTA. 2015; 19(36):229-240.

8. Freitas LJ. Uma interface Interacionista e Reflexiva do Ensino de Língua Portuguesa sob a Luz de Vygotsky e Habermas. Revista de Investigación en Ciencias Sociales y Humanidades. 2016 Jul 3(1).

9. Preto DR, Silva CA, Pereira LP, Costa MR, Cazella SC. Teorias de aprendizagem aplicadas à modalidade de educação a distância na saúde: uma revisão integrativa. Em Rede - Revista de Educação a Distância. 2017; 4(1).
10. Vygotsky LS. A formação social da mente. 4.ed. São Paulo: Martins Fontes, 1991.

11. Tibes CM, Dias JD, Westin, UM, Domingues, AN, Zem-Mascarenhas SH, Évora YDM. Development of digital educational resources for nursing education. Rev enferm UFPE on line. 2017. 11(Supl. 3):1326-34.

12. Tori R. Tecnologia e metodologia para uma educação sem distância. Em Rede Revista de Educação a distância. São Paulo, 2015; 2(2).

13. Domenico EBLD, Cohrs CR. Moodle platform for the construction of knowledge in intensive care: an experimental study. Acta Paul Enferm. 2016; 29(4):381-9.

14. Aredes NDA, Góes FSN, Silva MAI, Gonçalves MFC, Fonseca LMM. Objeto digital em enfermagem neonatal: impacto na aprendizagem de estudantes. Rev. Eletr. Enf. 2015 out./dez.17(4).

15. Rodrigues RCV, Peres HHC. An educational software development proposal for nursing in neonatal cardiopulmonary resuscitation. Rev Esc Enferm USP 2013; 47(1):235-41.

16. Morán J. Mudando a educação com metodologias ativas. Coleção Mídias Contemporâneas. Convergências Midiáticas, Educação e Cidadania: aproximações jovens. 2015; 2.

17. Vygotsky LV. Pensamento e linguagem. 3.ed. São Paulo: Martins Fontes, 1991.

18. Vygotsky LV. Pensamento e Linguagem. Rio de Janeiro: Martins Fontes, 1998.

19. Coelho L, Pisoni S. Vygotsky: Sua teoria e a influência na educação. Revista Pedfacos/cnecosório. 2012 ago, 2(1).

20. Thofehrn MB, Leopard MT. Construtivismo Sócio-Histórico de Vygostky e a Enfermagem. Revista 
Brasileira de Enfermagem. 2006 set-out. 59(5):694-8.

21. Góes, FSN, Camargo, RAA, Hara CYN, Fonseca LMM. Tecnologias educacionais digitais para educação profissional de nível médio em enfermagem. Rev. Eletr. Enf. 2014 abr/jun.16(2):453-61.

22. Vygotsky LS. A formação social da mente:

o desenvolvimento dos processos psicológicos. 6. Ed. São Paulo: Martins Fontes, 1996.

23. Salvador PTCO, Rodrigues CCFM, Lima KYN, Alves KYA, Santos VEP. Use and development of teaching technologies presented in nursing research. Rev Rene. 2015 maio-jun. 16(3):442-50. 